\title{
Cat Scratch Disease Complicated With Aseptic Meningitis and Neuroretinitis
}

Vitor Laerte Pinto Jr. ${ }^{1}$, André Land Curi ${ }^{1}$, Adriana da Silva Pinto ${ }^{1}$, Estevão Portela Nunes ${ }^{1}$, Maria de Lourdes Benamor Teixeira ${ }^{1}$, Tatiana Rozental ${ }^{2}$, Alexsandra Rodrigues Favacho ${ }^{2}$, Elba Regina Sampaio de Lemos ${ }^{2}$ and Márcio Neves Bóia ${ }^{2}$ ${ }^{1}$ Evandro Chagas Clinical Research Institute, Fiocruz; ${ }^{2}$ Oswaldo Cruz Institute, Fiocruz; Rio de Janeiro, RJ, Brazil

\begin{abstract}
Cat scratch disease (CSD) is a self limited condition characterized by fever, lymph node enlargement and less often eye involvement. Central nervous system involvement by Bartonella henselae infection is possibly an important cause of morbidity; its role as an agent of aseptic meningitis is unknown. We report a case of a 40 years-old man with CSD accompanied by aseptic meningitis and neuroretinitis. Serum indirect immmunofluorescence (IFI) assays for B. henselae were positive and the cerebrospinal fluid (CSF) analysis showed mononuclear pleocytosis and increased level of protein. Serological tests for other etiologies were negative. The patient responded well to antibiotic therapy with oral doxycicline plus rifampin and in the $12^{\text {th }}$ day of hospitalization evolved to total regression of the headache and partial regression of the visual loss. Clinicians should consider CSD as a differential diagnosis when assessing previously healthy patients with aseptic meningitis associated with regional lymphadenopathy and epidemiological history of feline contact.
\end{abstract}

Key-Words: Cat scratch disease, Bartonella henselae, aseptic meningitis.

Central nervous system involvement by Bartonella henselae infection is possibly an important cause of morbidity; its role as an agent of aseptic meningitis is unknown. So far only three cases have been described in the literature, two of them in patients with HIV infection $[1,2]$. We report a case of aseptic meningitis in a previously healthy patient, HIV negative, who also had visual loss due to neuroretinitis, a far more common complication of CSD.

Manifestations of CSD are well described and include self-limited course of fever along with lymph node enlargement linked epidemiologically with a history of intimate contact with cat, mainly scratch or bite. Generally children and young adults are more involved, but cases are seen in any age group [3]. Isolation of the causative agent is very difficult and in the past, therapy was initiated on clinical findings and epidemiological history criteria. Laboratory diagnosis is often retrospective, based mainly in serology and typical findings on histopathological examination of the affected lymph node [4]. Diagnosis of such infections is based on clinical information, histopathology, culture and serology. However, none of these methods alone is sufficiently sensitive or specific. In the past few years polymerase chain reaction (PCR) is emerging as a useful tool for specific and rapid diagnosis [5]. There is no therapeutic consensus on the need for, selection and duration of antimicrobial therapy. Bartonella spp. are susceptible to betalactams, rifampin, erythromycin and tetracyclines, and have variable susceptibility to clindamycin, quinolones and cotrimoxazol [6].

Received on 15 January 2008; revised 6 April 2008.

Address for correspondence: Dr. Vitor Laerte Pinto Jr. Av. Brasil n ${ }^{\circ}$ 4365. Zip code: 21040-360. Manguinhos, Rio de Janeiro, RJ, Brazil. Phone/fax: 55-21-3865-9571. E-mail: vitor.laerte@ipec.fiocruz.br.

The Brazilian Journal of Infectious Diseases 2008;12(2):158-160. (C) 2008 by The Brazilian Journal of Infectious Diseases and Contexto Publishing. All rights reserved.

\section{Case Report}

S.F.B. a 40 year-old man was referred to the inpatient service of Evandro Chagas Clinical Research Institute (which is dedicated to the research in infectious diseases), with a 15-day history of continuous headache, visual loss, right axilar lymph node enlargement and fever $\left(38.5^{\circ} \mathrm{C}\right)$. This patient referred that seven days before the beginning of symptoms he had been scratched on his right hand by a street cat. Physical examination revealed an enlarged, painful and mobile lymph node in the right axillary region (Figure 1). There was no residual lesion in the region of the scratch. No abdominal mass or signs of meningism were detected. Laboratory findings, including blood cell count, were normal. Serological tests for toxoplasmosis, CMV, syphilis, HIV, Hepatitis B and C and Sporothrix schenkii were all negative. Thoracic computed tomographyc scans revealed an enlarged lymph node in left axillary region measuring 3.6 x $3.2 \mathrm{~cm}$ with peripheral contrast enhancement. Brain computed tomography scans were normal. Fundus examination of the left eye revealed optic disc edema, perifoveal infiltrates and a stellate macular lesion (Figure 2). Azythromicin $500 \mathrm{mg}$ once daily was started.

On the fifth day of treatment the patient still referred headache and had persistence of fever; a lumbar puncture was done to investigate a meningeal involvement. CSF examination demonstrated a clear liquid containing 25 mononuclear cells per $\mathrm{mm}^{3}$, glucose level was $53 \mathrm{mg} / \mathrm{dL}$ and protein level was $97.4 \mathrm{mg} / \mathrm{dL}$. Based on these findings, antimicrobial treatment was changed to oral doxycicline (100 mg bid) associated with rifampin (300 mg bid).

Serology for Bartonella spp. was performed by indirect immunofluorescence assay, using commercial antigen, which revealed a 1:2048 immunoglobulin G (IgG) titer (cut-off 1:64); a second assay was done one week later resulting an IgG titer of 1:1024. Three more serum samples were analyzed and the fourth sample, collected 11 weeks after the first sample, showed IgG titer of 1:256. A sample of citrated whole blood collected 
Figure 1. Enlarged lymph node in the right axillary region.

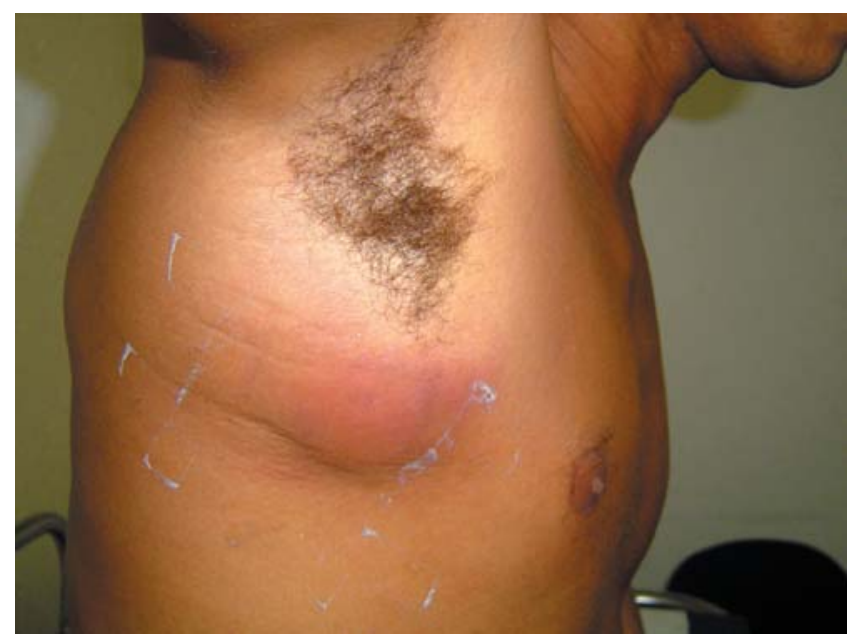

in the third day of hospitalization was subjected to PCR but was negative for Bartonella spp. Lymph node biopsy was not performed because the diagnosis was done based on clinical and epidemiology information and on typical ophthalmic alterations.

On the $12^{\text {th }}$ day of hospitalization there was an outstanding clinical response with total regression of the headache and partial regression of the visual loss. The patient was discharged and advised to follow-up in the Ophthalmology Outpatient Clinic. In the $30^{\text {th }}$ day of treatment there was total recovery of visual acuity and involution of the right axillary lymph node enlargement. Antimicrobial treatment was discontinued then.

\section{Discussion}

CSD is perhaps the most common cause of fever and lymphadenitis in children and young adults. In the United States the annual number of cases is estimated to be between 22,000 and 24,000 . It has been associated with exposure to cats in about $90 \%$ of cases [4,7]. The prevalence of antibodies against Bartonella henselae in the population in various studies ranged from $3 \%$ in EUA to $61 \%$ in Italy, even in those without previous evidence of CSD [8-10]. Epidemiological studies of Bartonella infection in Brazil suggests high seroprevalence but the clinical implications are not widely known [11,12].

Typical clinical manifestations usually begin after a cat scratch. A papule then pustule develops seven to 12 days at the inoculation site, followed by enlargement of lymph nodes one to three weeks later, which can persist for few weeks to several months. Low grade fever, malaise, headache are also commonly referred by the patients [13].

Neurological complications of CSD could be considered an emerging condition, being described each day a different pattern of disease [14-16]. The better described
Figure 2. Fundus photography showing hard exudates (circled area) and discrete disc edema.

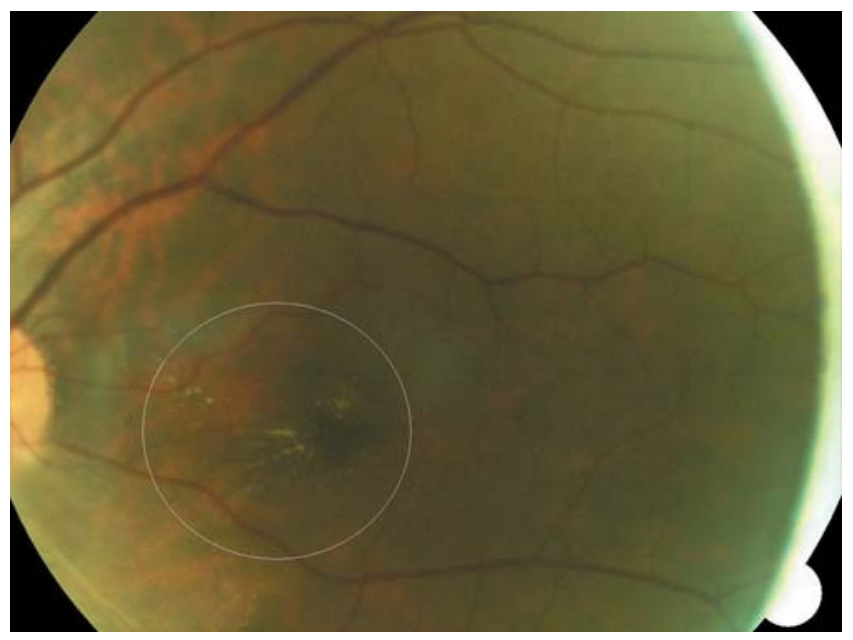

manifestation is encephalopathy occurring in $2 \%-3 \%$ of patients. A syndrome of aseptic meningitis is rarely described and we have found three cases in the literature, one reported by Lucey et al. in immunocompetent patient whose CSF analysis revealed 19 leukocytes $/ \mathrm{mm}^{3}$ (100\% mononuclear), a protein level of $62 \mathrm{mg} / \mathrm{dL}$, and a glucose level of $76 \mathrm{mg} / \mathrm{dL}$ [1]. The other two cases were reported by Wong et al. one in HIV-positive patient [2]. These findings are compatible with the alterations encountered in our patient and probably could be more often than is currently diagnosed.

Ocular involvement occurs in $5 \%$ to $10 \%$ of patients with CSD, what makes the eye the most commonly affected organ after the lymphatics [17]. The most frequently described manifestation is Parinaud oculoglandular syndrome, which is characterized by eye redness, foreign body sensation and epiphora [18]. In this case we could observe an optic neuropathy with optic disk swelling and the presence of a partial macular star. These alterations are denominated neuroretinitis and CSD is its most common cause. Very often patients also complain of visual loss, generally reversible with antibiotic treatment [19-21].

In relation to laboratory diagnosis, is important to remember that the differentiation among the species of Bartonella by serologic test is difficult and the characterization of Bartonella by molecular techniques should be also used as a confirmatory diagnostic technique, mainly in disseminated Bartonella infections.

Although the PCR has been negative and the diagnosis of this aseptic meningitis has been based on clinicalepidemiological information and serology, we concluded that the patient was probably infected by $B$. henselae. These findings highlight the importance of Bartonella infection as a neglected and emergent agent and the need for more clinical studies of this disease in Brazil. 


\section{References}

1. Lucey D., Dolan M.J., Moss C.W., et al. Relapsing illness due to Rochalimaea henselae in immunocompetent hosts: implication for therapy and new epidemiological associations. Clin Infect Dis 1992;14(3):683-8.

2. Wong M.T., Dolan M.J., Lattuada C.P., Jr., et al. Neuroretinitis, aseptic meningitis, and lymphadenitis associated with Bartonella (Rochalimaea) henselae infection in immunocompetent patients and patients infected with human immunodeficiency virus type 1. Clin Infect Dis 1995;21(2):352-60.

3. Adal K.A., Cockerell C.J., Petri W.A., Jr. Cat scratch disease, bacillary angiomatosis, and other infections due to Rochalimaea. N Engl J Med 1994;330(21):1509-15.

4. Manfredi R., Sabbatani S., Chiodo F. Bartonellosis: light and shadows in diagnostic and therapeutic issues. Clin Microbiol Infect 2005;11(3):167-9.

5. Hansmann Y., DeMartino S., Piemont Y., et al. Diagnosis of cat scratch disease with detection of Bartonella henselae by PCR: a study of patients with lymph node enlargement. J Clin Microbiol 2005;43(8):3800-6.

6. Rolain J.M., Brouqui P., Koehler J.E., et al. Recommendations for treatment of human infections caused by Bartonella species. Antimicrob Agents Chemother 2004;48(6):1921-33.

7. Chomel B.B., Boulouis H.J., Maruyama S., Breitschwerdt E.B. Bartonella spp. in pets and effect on human health. Emerg Infect Dis 2006;12(3):389-94.

8. Ferres G.M., Abarca V.K., Prado D.P., et al. Prevalence of Bartonella henselae antibodies in Chilean children, adolescents and veterinary workers. Rev Med Chil 2006;134(7):863-7.

9. Massei F., Messina F., Gori L., et al. High prevalence of antibodies to Bartonella henselae among Italian children without evidence of cat scratch disease. Clin Infect Dis 2004;38(1):145-8.

10. Tea A., Alexiou-Daniel S., Arvanitidou M., et al. Occurrence of Bartonella henselae and Bartonella quintana in a healthy Greek population. Am J Trop Med Hyg 2003;68(5):554-6.
11. da Costa P.S., Brigatte M.E., Greco D.B. Antibodies to Rickettsia rickettsii, Rickettsia typhi, Coxiella burnetii, Bartonella henselae, Bartonella quintana, and Ehrlichia chaffeensis among healthy population in Minas Gerais, Brazil. Mem Inst Oswaldo Cruz 2005;100(8):853-9.

12. Lamas C.C., Rozental T., Favacho A., et al. Bartonella sp. infections in HIV positive individuals in Rio de Janeiro, Brazil. In: $12^{\text {th }}$ International Congress on Infectious Diseases; 2006; Lisbon, Portugal.: Int J Infect Dis, 10(suppl 1): S176; 2006.

13. Dolan M.J., Wong M.T., Regnery R.L., et al. Syndrome of Rochalimaea henselae adenitis suggesting cat scratch disease. Ann Intern Med 1993;118(5):331-6.

14. Baylor P., Garoufi A., Karpathios T., et al. Transverse myelitis in 2 patients with Bartonella henselae infection (cat scratch disease). Clin Infect Dis 2007;45(4):e42-5.

15. Hmaimess G., Kadhim H., Saint Martin C., et al. Cat scratch disease presenting as meningomyeloradiculopathy. Arch Dis Child 2004;89(7):691-2.

16. Stockmeyer B., Schoerner C., Frangou P., et al. Chronic vasculitis and polyneuropathy due to infection with Bartonella henselae. Infection 2007;35(2):107-9.

17. Cunningham E.T., Koehler J.E. Ocular bartonellosis. Am J Ophthalmol 2000;130(3):340-9.

18. Carithers H.A. Cat-scratch disease. An overview based on a study of 1,200 patients. Am J Dis Child 1985;139(11):112433.

19. Ormerod L.D., Skolnick K.A., Menosky M.M., et al. Retinal and choroidal manifestations of cat-scratch disease. Ophthalmology 1998;105(6):1024-31.

20. Reed J.B., Scales D.K., Wong M.T., et al. Bartonella henselae neuroretinitis in cat scratch disease. Diagnosis, management, and sequelae. Ophthalmology 1998;105(3):459-66.

21. Solley W.A., Martin D.F., Newman N.J., et al. Cat scratch disease: posterior segment manifestations. Ophthalmology 1999;106(8):1546-53. 